\title{
Bounded Model Checking of Software using SMT Solvers instead of SAT Solvers
}

\author{
Alessandro Armando, Jacopo Mantovani, and Lorenzo Platania \\ Artificial Intelligence Laboratory \\ DIST, Università degli Studi di Genova \\ Viale F. Causa 13, 16145 Genova, Italy \\ \{armando, jacopo, lorenzo\}@dist.unige.it
}

\begin{abstract}
C Bounded Model Checking (CBMC) is one of the leading approaches to automatic software analysis. The key idea is to (i) build a propositional formula whose models correspond to program traces (of bounded length) that violate some given property and (ii) use state-ofthe-art SAT solvers to check the resulting formulae for satisfiability. In this paper we propose a generalisation of the CBMC approach based on an encoding into richer (but still decidable) theories than propositional logic. We show that our approach may lead to considerably more compact formulae than those obtained with CBMC. We have built a prototype implementation of our technique that uses a Satisfiability Modulo Theories (SMT) solver to solve the resulting formulae. Computer experiments indicate that our approach compares favourably with and on some significant problems outperforms CBMC.
\end{abstract}

\section{Introduction}

SAT-based Bounded Model Checking (BMC) [1] was originally proposed as a complementary technique to OBDD-based model checking for the automatic analysis of finite state systems (e.g. hardware circuits). The key idea is to encode bounded behaviours of the system that enjoy some given property as a propositional formula whose models (if any) describe a system trace leading to a violation of the property.

The application of Bounded Model Checking to software poses new challenges, as most programs are inherently infinite-state and new, non trivial issues such as the handling of (recursive) function calls and the modeling of complex data structures must be properly addressed. An elegant solution to the problem is proposed in $[2,3]$ and implemented in the CBMC (C Bounded Model Checking) model checker. The approach amounts to (i) building a propositional formula whose models correspond to program traces (of bounded length) violating some given property and (ii) using state-of-the-art SAT solvers to check the resulting formulae for satisfiability.

In this paper we propose a generalisation of the CBMC approach. Instead of encoding the program into a propositional formula, we encode it into a quantifierfree formula to be checked for satisfiability w.r.t. some given decidable theory 
(henceforth called background theory) and use a state-of-the-art SMT (Satisfiability Modulo Theories) solver to perform the satisfiability checking.

We show that our approach may lead to considerably more compact formulae when arrays are involved in the input program. In particular the size of the formulae generated by our approach does not depend on the size of the bit-vector representation of the basic data types nor on the size of the arrays occurring in the program, whereas the encoding technique implemented in CBMC depends on both. Experimental results obtained with a prototype implementation of our technique, called SMT-CBMC, confirm the effectiveness of our approach: SMTCBMC scales significantly better than CBMC on a number of problems involving complex interactions of arithmetics and arrays manipulation as the size of the arrays occurring in the input program increases.

Structure of the paper. In the next section (Section 2) we provide a brief introduction to SMT and present a set of decidable theories that we will refer to in the rest of the paper. In Section 3 we present our generalisation to the CBMC approach: we describe the generation of the formula, the different approaches to solve the formula, and how error traces are reconstructed by exploiting the information returned by the SMT solver. In Section 4 we describe our prototype tool SMT-CBMC and present the experimental results. In Section 5 we discuss the related work and finally, in Section 6, we draw some concluding remarks.

\section{Satisfiability Modulo Theories}

Given a decidable theory $\mathcal{T}$ and a quantifier-free formula $\phi$ in the same language as $\mathcal{T}$, we say that $\phi$ is $\mathcal{T}$-satisfiable if and only if there exists a model of $\mathcal{T}$ which is also a model of $\phi$ or, equivalently, if $\mathcal{T} \cup\{\phi\}$ is satisfiable. An SMT solver for $\mathcal{T}$ is a program capable to determining the $\mathcal{T}$-satisfiability of every quantifier-free formula $\phi$ in the same language as $\mathcal{T}$. Let $\Gamma \cup\{\phi\}$ be a set of formulae in the same language as $\mathcal{T}$, we say that $\phi$ is a $\mathcal{T}$-consequence of $\Gamma$, in symbols $\Gamma \models_{\mathcal{T}} \phi$, if and only if every models of $\mathcal{T} \cup \Gamma$ is a model of $\phi$. Obviously, the problem of determining whether $\mathcal{C} \models_{\mathcal{T}} \phi$ holds can be reduced to the problem of checking the $\mathcal{T}$-satisfiability of $\mathcal{C} \cup\{\neg \phi\}$.

Over the last three decades, a great deal of attention has been paid to solving the SMT problem for a number of (decidable) theories of interest such as, e.g., Linear Arithmetics, the theory of lists, the theory of arrays, and-more recently - the theory of bit-vectors. The practical relevance of these theories in verification cannot be overestimated as arithmetics, lists, arrays, and bit-vectors are ubiquitous in Computer Science. Moreover, since these entities rarely occur in isolation, the problem of building SMT solvers for the combination of two (or more) decidable theories (say $\mathcal{T}_{1} \cup \mathcal{T}_{2}$ ) out of SMT solvers for the component theories (say $\mathcal{T}_{1}$ and $\mathcal{T}_{2}$ ) has also been thoroughly investigated and solutions identified $[4,5]$. More recently the problem of combining the effectiveness of state-of-theart SAT solvers with SMT solvers has received growing attention and has led to a new generation of SMT solvers capable of remarkable performance [6]. 
In the rest of this section we give a brief description of the decidable theories that are relevant for the present paper.

Linear Arithmetics. By Linear Arithmetics we mean standard arithmetics (either over $\mathbb{Z}, \mathbb{Q}$, or $\mathbb{R}$ ) with addition (i.e. + ) and the usual relational operators (e.g. =, $<, \leq,>, \geq$ ) but without multiplication. Multiplication by a constant, say $n * x$ where $n$ is a numeral, is usually allowed but it is just a notational shorthand for the (linear) expression $x+\cdots+x$ with $n$ occurrences of the variable $x$.

The theory of arrays. Arrays are data structures representing arbitrary associations of elements to a set of indexes. Unlike arrays available in standard programming languages, the arrays modelled by the theory of arrays need not to have finite size. Given sorts INDEX, ELEM and ARRAY for indices, elements, and arrays (resp.) and function symbols select : ARRAY $\times$ INDEX $\rightarrow$ ELEM and store : ARRAY $\times$ INDEX $\times$ ELEM $\rightarrow$ ARRAY, the standard presentation of the theory of arrays consists of the following two axioms:

$$
\begin{array}{cc}
\forall a, i, e . & \operatorname{select}(\operatorname{store}(a, i, e), i)=e \\
\forall a, i, j, e . \quad(i \neq j \supset \operatorname{select}(\operatorname{store}(a, i, e), j)=\operatorname{select}(a, j))
\end{array}
$$

with variable $a$ of sort ARRAY, $i$ and $j$ of sort INDEX, and $e$ of sort ELEM.

SMT solvers for the theory of arrays are described in $[7,8]$.

The theory of records. Records are data structures that aggregate attribute-value pairs. Let $I d=\left\{i d_{1}, \ldots, i d_{n}\right\}$ be a set of field identifiers and $\mathrm{T}_{1}, \ldots, \mathrm{T}_{n}$ be types, $\operatorname{REC}\left(i d_{1}: \mathrm{T}_{1}, \ldots, i d_{n}: \mathrm{T}_{n}\right)$, henceforth abbreviated REC, is the sort of records that associate an element of type $\mathrm{T}_{k}$ to the field identifier $i d_{k}$, for $k=1, \ldots, n$. The signature of the theory of records consists of a pair of function symbols rselect $_{k}: \operatorname{REC} \rightarrow \mathrm{T}_{k}$ and rstore $_{k}: \operatorname{REC} \times \mathrm{T}_{k} \rightarrow \operatorname{REC}$ for $k=1, \ldots, n$. The theory is finitely presented by the following axioms:

$$
\begin{array}{ll}
\forall r, e . \quad \operatorname{rselect}_{k}\left(\operatorname{rstore}_{k}(r, e)\right)=e \quad \text { for } k=1, \ldots, n \\
\forall r, e . \operatorname{rselect}_{l}\left(\operatorname{rstore}_{k}(r, e)\right)=\operatorname{rselect}_{l}(r) & \text { for } k, l \text { such that } 1 \leq k \neq l \leq n
\end{array}
$$

where $r$ has sort REC and $e$ has sort $\mathrm{T}_{i}$.

A SMT solver for the theory of records is described in [9].

The theory of bit-vectors. Similarly to arrays, bit-vectors associate elements to a set of indexes, but unlike arrays the set of indexes is finite. Moreover the element associated to each index is boolean valued. Many theories of bit-vectors have been proposed in the literature [10-14], the main difference being whether bit-vectors are allowed to have variable size or not. For our purposes, the theory of fixed-size bit-vectors does suffice. The theory we consider has a sort $\operatorname{BV}(n)$ for each positive integer $n$ and a rich family of functions symbols consisting of

- word-level functions, e.g. - $[i: j]: \mathrm{BV}(m) \rightarrow \mathrm{BV}(j-i+1)$ (bit-vector extraction) for $0 \leq i \leq j \leq m$, @ : $\mathrm{BV}(m) \times \mathrm{BV}(n) \rightarrow \mathrm{BV}(m+n)$ (bit-vector concatenation) for $m, n>0$; 
- bitwise functions, e.g. ${ }^{\sim}: \operatorname{BV}(n) \rightarrow \operatorname{BV}(n)$ (bitwise not), \& $: \operatorname{BV}(n) \times \operatorname{BV}(n) \rightarrow$ $\mathrm{BV}(n)$ (bitwise and), $\mid: \operatorname{BV}(n) \times \mathrm{BV}(n) \rightarrow \mathrm{BV}(n)$ (bitwise or) for $n>0$;

- arithmetic functions, e.g. $+: \mathrm{BV}(n) \times \mathrm{BV}(n) \rightarrow \mathrm{BV}(n)$ (addition modulo $2^{n}$ ) for $n>0$.

\section{Bounded Model Checking of Sequential Software}

As in [2], preliminarily to the generation of the formula, we preprocess the input program (Section 3.1). Given a bound $n>0$, this amounts to applying a number of transformations which lead to a simplified program whose execution traces have finite length and correspond to the (possibly truncated) traces of the original program. The quantifier-free formula is then obtained by generating a quantifier-free formula for each statement of the resulting program (Section 3.2) and the resulting formula is fed to a SMT solver (Section 3.3). If an execution path leading to a violation of an assert statement occurring in the original program is detected, then a corresponding trace is built and returned to the user for inspection (Section 3.4).

In order to simplify the presentation, we assume that $=$ is the only assignment operator occurring in the program and that no pointer variables nor conditional expressions occur in the program. Notice that all these simplifying assumptions can be readily lifted as in [15].

\subsection{The Preprocessing Phase}

The preprocessing activity starts by replacing break and continue statements with semantically equivalent goto statements. The switch construct is replaced by a proper combination of if and goto statements. Loops are then unwound by reducing them to a sequence of nested if statements. For instance, while loops are removed by applying the following transformation $n$ times:

$$
\operatorname{while}(e)\{I\} \longrightarrow \operatorname{if}(e)\{I \operatorname{while}(e)\{I\}\}
$$

The last while loop is finally replaced by the statement assert $(! e)$; , called unwinding assertion. The failure of an unwinding assertion indicates that the bound $n$ is not sufficient to model the system and the properties entirely, and that $n$ must be increased.

Non recursive functions are then inlined. Recursive function calls and backward goto's are unwound similarly to loop statements. Forward goto statements are transformed into equivalent if statements as explained in [1].

Next we put the program in if normal form by invoking the normalisation algorithm of Fig. 1 with $G=\emptyset$. This normalisation step removes the else constructs and pushes the if statements downwards in the abstract syntax tree of the program till they are applied to atomic statements only. An example of the transformation of a program in if normal form is given in Fig. 2.

Notice that a program in if normal form is a sequence of statements of the form if $(c) s$; where $s$ is either an assignment or an assert statement. 


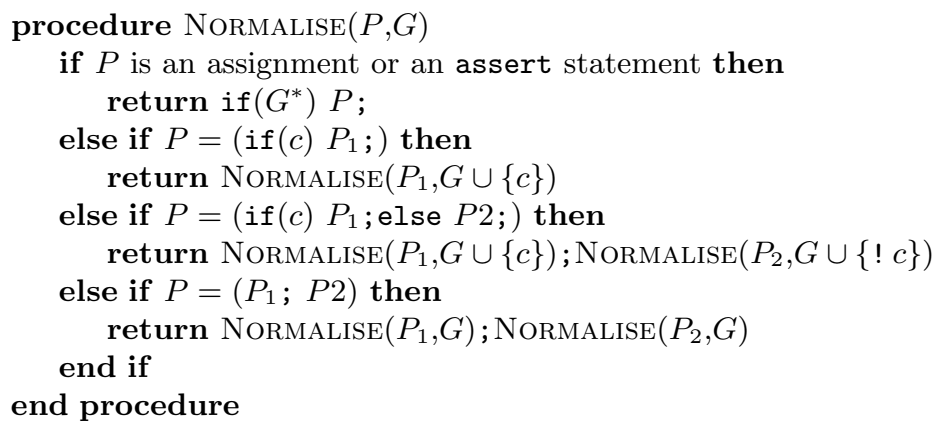

Legenda: If $G$ is a finite set of expressions then $G^{*}$ is an expression defined as follows: $\emptyset^{*}=$ true, $\{c\}^{*}=c$, and $\left\{c_{1}, \ldots, c_{n}\right\}^{*}=\left(c_{1} \& \& \cdots \& \& c_{n}\right)$ if $n \geq 2$.

Fig. 1. Turning the program in if normal form

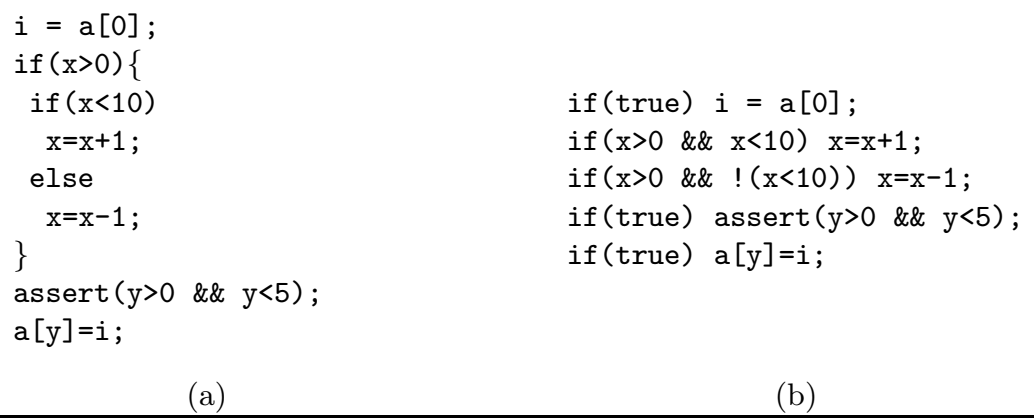

(a)

(b)

Fig. 2. Turning a program in if normal form: (a) the original program and (b) the normalised program. 


\subsection{The Encoding Phase}

The application of the previous transformations leaves us with a program $P$ in if normal form. We now show how to build two sets of quantifier-free formulae $\mathcal{C}$ and $\mathcal{P}$ such that $\mathcal{C} \models_{\mathcal{T}} \wedge \mathcal{P}$ for some given background theory $\mathcal{T}$ if and only if no computation path of $P$ violates any assert statement in $P$.

Let $v$ be a program variable and $i$ a program location. We define $\alpha(v, i)$ to be the number of assignments made to $v$ prior to location $i$. For each statement in $P$ at location $i$ of the form if $(c) v=e ; \mathcal{C}$ contains a formula of the form: ${ }^{1}$

$$
v_{\alpha(v, i)+1}=\left(c^{\prime} ? e^{\prime}: v_{\alpha(v, i)}\right)
$$

where $c^{\prime}$ and $e^{\prime}$ are obtained from $c$ and $e$ respectively by replacing every variable $w$ with $w_{\alpha(w, i)}$ and every expression of the form $a[e]$ with select $\left(a_{\alpha(a, j)}, e\right)$. Similarly, for each statement in $P$ at location $i$ of the form if $(c) a\left[e_{1}\right]=e_{2} ; \mathcal{C}$ contains a formula of the form:

$$
a_{\alpha(a, i)+1}=\left(c^{\prime} ? \operatorname{store}\left(a_{\alpha(a, i)}, e_{1}^{\prime}, e_{2}^{\prime}\right): a_{\alpha(a, i)}\right)
$$

where $c^{\prime}, e_{1}^{\prime}$, and $e_{2}^{\prime}$ are obtained from $c, e_{1}$, and $e_{2}$ respectively by substituting every variable $w$ with $w_{\alpha(w, i)}$ and every expression of the form $a[e]$ with $\operatorname{select}\left(a_{\alpha(a, j)}, e\right)$.

The set $\mathcal{P}$ contains a formula of the form $\left(c^{\prime} \supset e^{\prime}\right)$ for each statement in $P$ at location $i$ of the form if $(c)$ assert $(e)$; , where $c^{\prime}$ and $e^{\prime}$ are obtained from $c$ and $e$ respectively by replacing every variable $w$ with $w_{\alpha(w, i)}$ and every expression of the form $a[e]$ with $\operatorname{select}\left(a_{\alpha(a, j)}, e\right)$.

$$
\begin{aligned}
\mathcal{C}=\{ & i_{1}=\left(\text { TRUE } ? \text { select }\left(a_{0}, 0\right): i_{0}\right), \\
& x_{1}=\left(\left(x_{0}>0 \wedge x_{0}<10\right) ? x_{0}+1: x_{0}\right), \\
& x_{2}=\left(\left(x_{1}>0 \wedge \neg\left(x_{1}<10\right)\right) ? x_{1}-1: x_{1}\right), \\
& \left.a_{2}=\left(\text { TRUE } ? \text { store }\left(a_{1}, y_{0}, i_{1}\right): a_{1}\right)\right\} \\
\mathcal{P}= & \left\{\text { TRUe } \supset\left(y_{0}>0 \wedge y_{0}<5\right)\right\}
\end{aligned}
$$

Fig. 3. The sets of formulae $\mathcal{C}$ and $\mathcal{P}$ for the program in Fig. 2.

\subsection{Solving the Formula}

Solving the Formula with a SAT Solver. In [2] this problem is reduced to a propositional satisfiability problem which is then fed to the Chaff SAT

\footnotetext{
${ }^{1}$ We use the expression $v=\left(c ? e_{1}: e_{2}\right)$ as an abbreviation for the formula $(c \supset v=$ $\left.e_{1}\right) \wedge\left(\neg c \supset v=e_{2}\right)$.
} 
solver [16]. This is done by modeling variables of basic data types (e.g. int and float) as fixed-size bit-vectors and by considering the equations in $\mathcal{C}$ and in $\mathcal{P}$ as bit-vectors equations. Each array variable $a$ is also replaced by $\operatorname{dim}(a)$ distinct variables $a^{0}, \ldots, a^{\operatorname{dim}(a)-1}$ and each formula of the form $(1)$ occurring in $\mathcal{C}$ is replaced by the formula

$$
\bigwedge_{i=0}^{\operatorname{dim}(a)-1} a_{k}^{i}=\left(\left(c \wedge e_{1}=i\right) ? e_{2}: a_{k-1}^{i}\right)
$$

Finally each term of the form select $\left(a_{\alpha(a, j)}, e\right)$ is replaced by a new variable, say $x$, and the following formulae are added to $\mathcal{C}$

$$
\bigwedge_{i=0}^{\operatorname{dim}(a)-1}\left((e=i) \supset x=a_{\alpha(a, j)}^{i}\right)
$$

The resulting set of bit-vector equations are then turned into a propositional formula. Variables of struct types are treated in a similar way. Notice that the size of the propositional formula generated in this way depends ( $i$ ) on the size of the bit-vector representation of the basic data types as well as (ii) on the size of the arrays used in the program. It is worth pointing out that if the program contains a multi-dimensional array $a$ with dimensions $d_{1}, \ldots, d_{m}$, then the number of added formulae grows as $O\left(d_{1} \cdot d_{2} \cdot \ldots \cdot d_{m}\right)$.

Solving the Formula with a SMT Solver. The alternative approach proposed in this paper is to use a SMT solver to directly check whether $\mathcal{C} \models_{\mathcal{T}} \wedge \mathcal{P}$. By proceeding in this way the size of the formula given as input to the SMT solver does not depend on the size of the bit-vector representation of the basic data types nor on the size of the arrays occurring in the program. ${ }^{2}$ Moreover the use of a SMT solver gives us additional freedom in the way we model the basic data types. In fact, program variables with numeric type (e.g. int, float) can be modelled by variables ranging over bit-vectors or over the corresponding numerical domain (e.g. $\mathbb{Z}, \mathbb{R}$, resp.). If the modeling of numeric variables is done through fixed-size bit-vectors, then the result of the analysis is precise but it depends on the specific size considered for the bit-vectors. If, instead, the modelling of numeric variables is done through the corresponding numerical domain, then the result of the analysis is independent from the actual binary representation, but this comes to the price of loosing completeness of the analysis if non linear expressions occur in the program.

\footnotetext{
${ }^{2}$ It must be said that SMT solvers for the theory of bit-vectors may expand parts of the the formula by a technique known as bit-blasting, however this is usually done as a last resort and in many cases higher level and less expensive techniques are enough to solve the problem at hand [14].
} 


\subsection{Building the Error Trace}

Whenever CVC Lite is asked to determine whether $\Gamma \models_{\mathcal{T}} \phi$, but this does not hold, the tool returns a finite set of formulae $\mathcal{K}$ such that $\Gamma, \mathcal{K} \models_{\mathcal{T}} \neg \phi$. The set of formulae $\mathcal{K}$ is said to be a counterexample for $\Gamma \models_{\mathcal{T}} \phi$.

Let $\mathcal{K}$ be a counterexample for $\mathcal{C} \models_{\mathcal{T}} \wedge \mathcal{P}$. We have defined a procedure that builds an error trace witnessing the violation of an assert statement occurring in the program $P$. The procedure (shown in Figure 4) traverses the control flow graph $G_{P}$ of $P$ starting from the first statement of $P$. Whenever a conditional statement if $(e)$ is met, then the "then" branch is taken if $\mathcal{C}, \mathcal{K}=\mathcal{T}$ e. If instead $\mathcal{C}, \mathcal{K} \models_{\mathcal{T}} \neg e$, then the "else" branch is taken. The control flow graph $G_{P}$ of $P$ is a directed graph $G_{P}=\left(N_{P}, \operatorname{Succ}_{P}\right)$, where $N_{P}=\{1, \ldots, n\}$ is the set of vertices and $\operatorname{Succ}_{P}: N_{P} \rightarrow 2^{N_{P}}$ maps each vertex in the set of its successors. For every vertex $i$ such that $1 \leq i \leq n, s_{i}$ denotes the program statement corresponding to $i$. By convention, node 1 of $G_{P}$ denotes the first statement of $P$ to be executed. If $s_{i}$ is a conditional statement (i.e. it is of the form if $(e)$ ), then $\operatorname{Succ}_{P}(i)=\left\{\operatorname{Tsucc}_{P}(i), F_{\operatorname{succ}_{P}}(i)\right\}$, where $T \operatorname{succ}_{P}(i)\left(F_{\operatorname{succ}_{P}}(i)\right)$ denotes the successor of $i$ when $e$ evaluates to true (false, resp.). If $s_{i}$ is an assignment or an assertion statement, $\operatorname{Succ}_{P}(i)=\{j\}$, with $j \in N_{P}$, and we define $\operatorname{sSucc}_{P}(i)=j$.

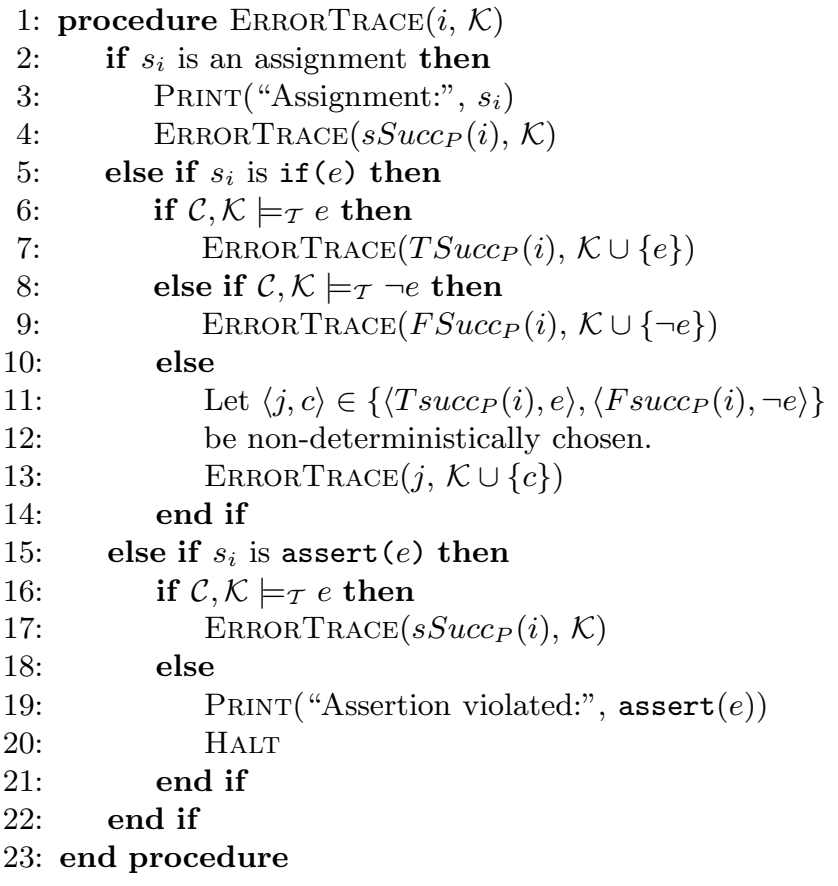

Fig. 4. Building the program trace. 
Notice that lines 11-13 of the algorithm allow for the non deterministic selection of a branch of a conditional statement if neither $\mathcal{C}, \mathcal{K} \models_{\mathcal{T}} e$ nor $\mathcal{C}, \mathcal{K} \models_{\mathcal{T}} \neg e$ hold. This is necessary because the counterexample $\mathcal{K}$ might not be sufficient to determine the branch to choose. In this event, the branch can be chosen non deterministically. Notice that this is a form of "don't care" non-determinism and therefore no backtracking is necessary. As an example of this, it can be noted that in the program of Figure 2 the assertion is violated independently from the value of variables $\mathrm{x}$ and $i$, and therefore also independently from the choice of the branches of the if statements. In fact, CVC Lite outputs a counterexample $\mathcal{K}=\left\{y_{0} \geq 5\right\}$ for which neither $\mathcal{C}, \mathcal{K} \models \mathcal{T}\left(x_{0}>0 \wedge x_{0}<10\right)$ nor $\mathcal{C}, \mathcal{K} \models_{\mathcal{T}}\left(x_{0}>0 \wedge \neg\left(x_{0}<10\right)\right)$ hold.

\section{Experimental Results}

In order to assess the effectiveness of our approach we have developed a prototype implementation called SMT-CBMC. SMT-CBMC consists of four main modules, implemented in about 5,000 lines of Prolog code. The first module parses the input program, the second carries out the preprocessing, the third builds the quantifier-free formula, and the fourth module solves the formula according to the user options by invoking CVC Lite. ${ }^{3}$ The latter module also builds and prints the error trace whenever a counterexample is returned by CVC Lite.

We have run SMT-CBMC against a number of families of $\mathrm{C}$ programs. Each family of programs is parametric in a positive integer $N$ and such that both the size of the arrays occurring in the programs and the number of iterations done by the programs depend on $N$. Therefore the instances become harder as the value of $N$ increases. The benchmark problems considered are:

- BubbleSort.c $(N)$, an implementation of the Bubble Sort algorithm [17],

- SelectSort.c $(N)$, an implementation of the Selection Sort algorithm [17],

- BellmanFord.c $(N)$, an implementation of the Bellman Ford algorithm [18, $19]$ for computing single-source shortest paths in a weighted graph, and

- Prim.c $(N)$ an implementation of Prim's algorithm [20] for finding a minimum spanning tree for a connected weighted graph.

Notice that these programs are well-known and therefore the result of the analysis is not interesting in itself. However they allow us to carry out a systematic and quantitave assessment of the tools as the size of the arrays involved in the programs increases. It is also worth pointing out that all the benchmark problems considered involve a tight interplay between arithmetics and array manipulation.

We have run both SMT-CBMC and CBMC on our benchmark programs. We report the total time spent by the tools to tackle each individual instance considered and, when relevant, also the time spent by CVC Lite to solve the formula generated by SMT-CBMC. Times are in seconds. All experiments have been obtained on a Pentium IV machine running Linux with the memory limit set to

${ }^{3}$ Currently SMT-CBMC can represent numeric data types with corresponding numeric domains as well as with fixed-size bit-vectors. 
$800 \mathrm{MB}$ and the time limit set to 30 minutes. CBMC has been invoked by manually setting the unwinding bound (CBMC --unwind $\mathrm{n}$ option) and by disabling simplification (CBMC --no-simplify option). ${ }^{4}$

All the experiments presented in the rest of this section have been obtained by modeling the basic data types using bit-vectors thereby enabling the decision procedure for the theory of bit-vectors available in CVC Lite during the solving phase. Experimental results indicate that similar performances are obtained by modeling the numerical variables with the integers thereby enabling the decision procedure for linear arithmetics available in CVC Lite during the solving phase.

More information about the experiments is available at URL http://www . ai.dist.unige.it/eureka.

\subsection{Sorting algorithms}

The Bubble Sort algorithm (see Figure 5) sorts the array a by using two nested loops that repeatedly swap adjacent elements. The assertion statements at the end of the program check that the array has been sorted. The parameter $N$ here determines the size of the array, as well as the number of unwindings for each loop. Notice that in this case the number of unwindings grows quadratically with $N$ as there are two nested loops.

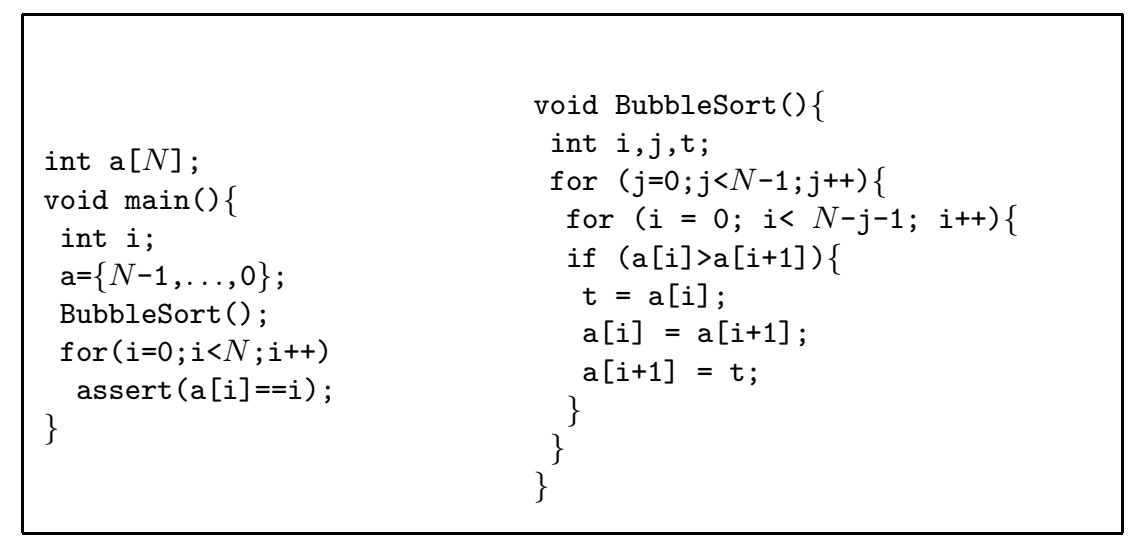

Fig. 5. Source code of BubbleSort.c $(N)$

The experimental results obtained for this family of programs are given in Figure 6. Plot (a) shows the time spent by the tools in analysing the program

\footnotetext{
${ }^{4}$ It is worth pointing out that CBMC features also an (undocumented) option --cvc whose effect is to output the bit-vector equations of the formula in the CVC format [21]. In this way it is possible to reason at the word-level, but still not using the theory of arrays. However this option is still experimental and not yet fully operational and therefore we have been unable to carry out experiments with it.
} 
while plot (b) shows the size (in bytes) of the encodings. In both cases the value of $N$ is on the the $x$-axis. CBMC runs out of memory for $N=17$, while

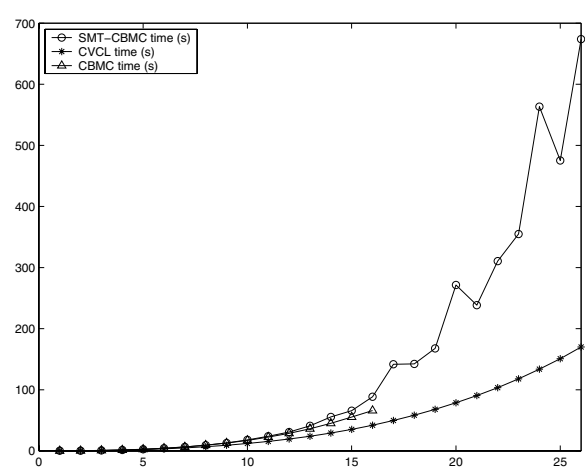

(a) Time spent by the tools

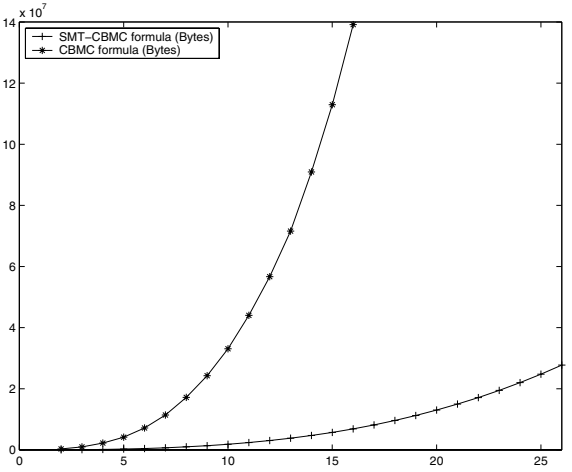

(b) Size of the formulae

Fig. 6. Results on BubbleSort.c $(N)$

SMT-CBMC can still analyse programs for $N=26$. A comparison between the formulae sizes of SMT-CBMC and CBMC substantiates our remarks about the size of the encodings: the formula built by CBMC for $N=16$ is roughly one order of magnitude bigger than the one built by SMT-CBMC.

Similarly to Bubble Sort, Selection Sort (see Figure 7) counts two nested loops and a swap operation, and the assertions at the end of the program check that the given array has been sorted. Unlike Bubble Sort, where the swap is guarded by an if within the nested loop, the swap operation is done $N$ times, where $N$ is the size of the array, without any guard. Therefore, the encoding grows as $O(N \cdot \operatorname{dim}(a))$, where $\operatorname{dim}(a)$ is the size of the array. As shown in plot b of Figure 8), CBMC runs out of memory already for $N=21$, whereas SMT-CBMC analyses instances till $N=29$.

\subsection{The Bellman-Ford algorithm}

The problems of the $\operatorname{BellmanFord}(N)$ family model are implementations of the Bellman Ford algorithm with a graph comprising 5 nodes and $N$ (randomly generated) edges. Each edge is associated with a (randomly generated) positive weight. The instance for $N=5$ is given in Fig. 9. The edges are represented by the arrays Source and Dest, the weights by the array Weight. The assert statements at the end on the program check that all the paths originating from the source node (represented by 0 ) have positive weight.

The results of the experiments are given in the plots of Fig. 10, where the $x$ axis represents the number of edges. Notice that the maximum value for $N$ is 20 in this case as the maximum number of edges in a fully connected directed 


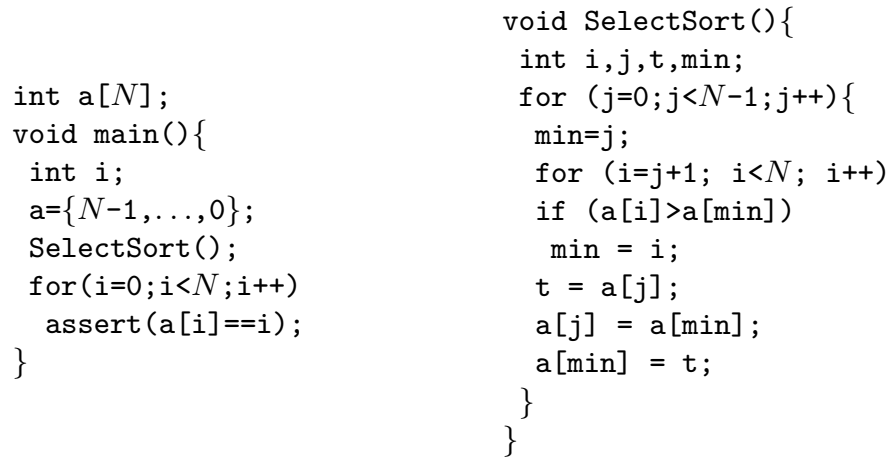

Fig. 7. Source code of SelectSort.c $(N)$

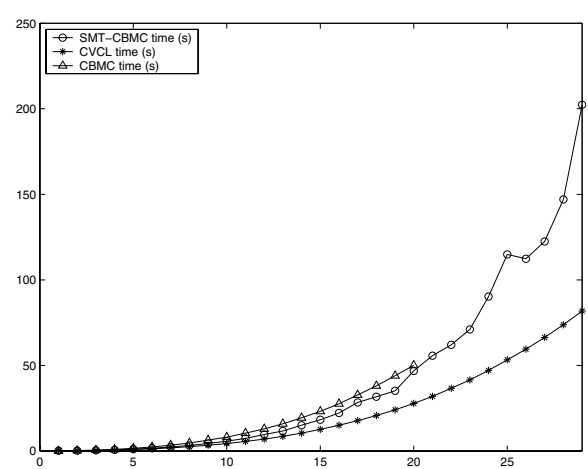

(a) Time (seconds) spent by the tools

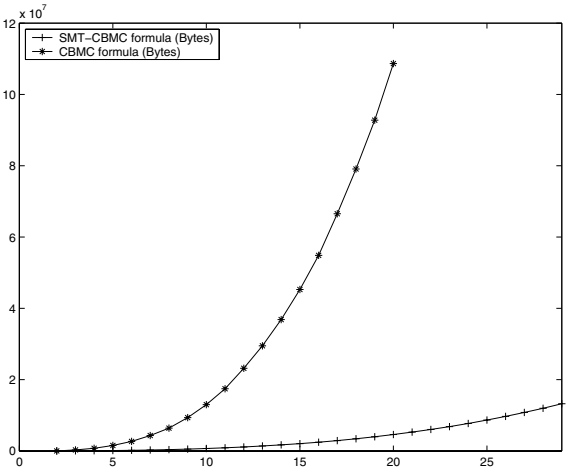

(b) Size of the formulae

Fig. 8. Results on SelectSort.c $(N)$

graph is $k(k-1)$ where $k$ is the number of nodes. Plot (a) displays the time spent by the tools in analysing the problems while plot (b) shows the size of the formulae. Notice that the formula generated by CBMC is already one order of magnitude bigger than the one of SMT-CBMC, for $N=12$.

\subsection{Prim's algorithm}

Prim's algorithm [20] finds a minimum spanning tree for a connected weighted graph. As in the Bellman-Ford implementation (Fig. 9), three arrays are used to model the attributes of the edges that connect the nodes of the graph. We used instances where the number of nodes of the graph is set to 4 and the number of edges increases according to the parameter $N$, starting from $N=4$. 


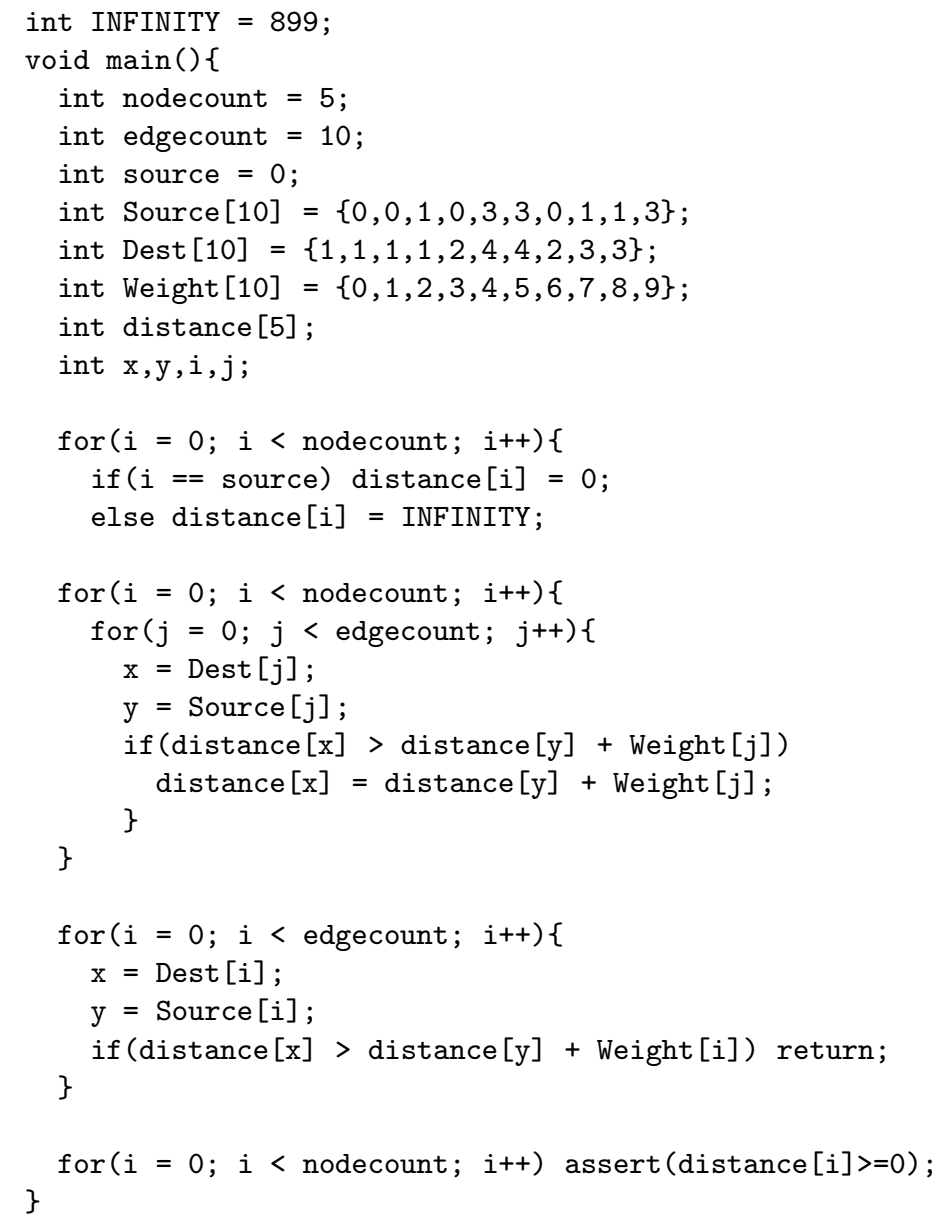

Fig. 9. Source code of the instance of $\operatorname{BellmanFord}(N)$ for $N=10$.

As shown in Fig. 1, already for $N=4$ the size of the formula output by CBMC is roughly 14 times bigger than the one of SMT-CBMC. For $N=7$ the difference becomes greater: the formula generated by CBMC becomes roughly 21 times bigger than the one of SMT-CBMC. For $N=8$, SMT-CBMC analyses the input program generating a formula of almost $8 \mathrm{MB}$, while CBMC runs out of memory.

\section{Related Work}

In the recent years a number of Model Checking procedures and tools have been developed for the verification of sequential software. 


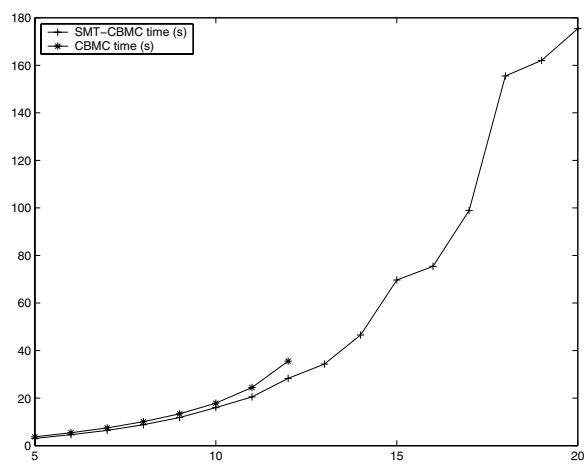

(a) Time spent by the tools

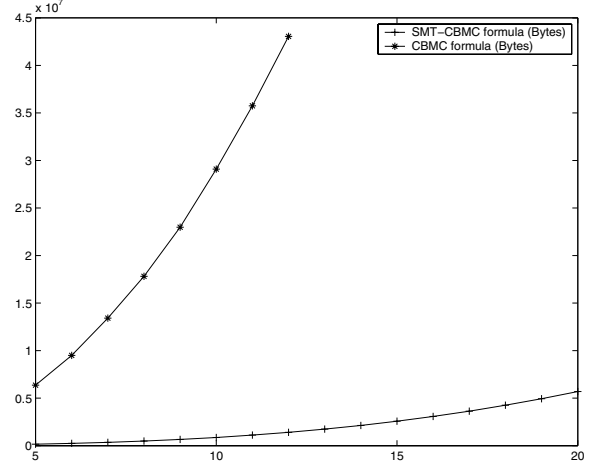

(b) Size of the formulae

Fig. 10. Results on BellmanFord.c $(N)$

Table 1. Size (in bytes) of the formulae generated by SMT-CBMC and CBMC for $\operatorname{Prim}(N)$.

\begin{tabular}{|c|c|r|}
\hline$N$ & SMT-CBMC & \multicolumn{1}{|c|}{ CBMC } \\
\hline \hline 4 & $1,108,211$ & $14,269,837$ \\
5 & $1,460,245$ & $31,453,119$ \\
6 & $2,755,215$ & $59,675,705$ \\
7 & $4,761,352$ & $108,045,992$ \\
8 & $7,704,875$ & memory out \\
\hline
\end{tabular}

SLAM [22], BLAST [23], and MAGIC [24] extend a symbolic model checking procedure for boolean programs with abstraction and refinement. Their approach has been shown to be very effective on specific application domains such as device drivers programming. However, when they come to reason about arrays they trade precision for efficiency. For instance SLAM and BLAST do not distinguish different elements of an array and this may lead them to report unsound results.

Saturn [25] is an efficient software error-detection tool that, like CBMC, translates C programs into boolean formulae that are then fed to a SAT solver. One of the distinguishing features of Saturn w.r.t. CBMC is the computation of summaries for each analysed function in order to speed up the (interprocedural) analysis. But again efficiency is obtained at the cost of loosing soundness: similarly to SLAM and BLAST, Saturn does not distinguish different elements of an array.

Both CBMC and SMT-CBMC treat arrays in a precise way, but they only consider execution traces of bounded length, limitation that can be mitigated by iterating the technique for increasing values of the unwinding bound. As shown in Section 4 SMT-CBMC can be considerably more effective than CBMC when applied to programs involving arrays of non-negligible size. However when 
no arrays occur in the program or when the arrays have small size CBMC can be more effective than SMT-CBMC. This suggests that the compilation to SMT should be seen as a complement and not as an alternative to the compilation to SAT. An interesting point is to determine syntactic criteria that allow us to determine for any given program which of the two encoding techniques is likely to perform best.

\section{Conclusion}

We have presented a Bounded Model Checking technique for sequential programs which uses SMT solvers instead of SAT solvers. Our work generalises the one presented in [2] and we have shown that our encoding technique generates considerably more compact formulae than CBMC when arrays are involved in the input program. In particular the size of the formulae generated by our approach does not depend on the size of the bit-vector representation of the basic data types nor on the size of the arrays occurring in the program.

Experimental results confirm the effectiveness of our approach: on problems involving complex interactions of arithmetics and arrays manipulation SMTCBMC scales significantly better than CBMC as the size of the arrays occurring in the input program increases.

\section{References}

1. Biere, A., Cimatti, A., Clarke, E.M., Zhu, Y.: Symbolic model checking without bdds. In Cleaveland, R., ed.: Proceedings of TACAS. Volume 1579 of Lecture Notes in Computer Science., Springer (1999) 193-207

2. Kroening, D., Clarke, E., Yorav, K.: Behavioral consistency of C and Verilog programs using bounded model checking. In: Proceedings of DAC, ACM Press (2003) 368-371

3. Clarke, E., Kroening, D., Lerda, F.: A tool for checking ANSI-C programs. In Jensen, K., Podelski, A., eds.: TACAS. Volume 2988 of LNCS., Springer (2004) 168-176

4. Nelson, G., Oppen, D.: Simplification by Cooperating Decision Procedures. ACM Transactions on Programming Languages and Systems 1 (1979) 245-257

5. Shostak, R.E.: Deciding Combinations of Theories. J. ACM 31 (1984) 1-12

6. Barrett, C., de Moura, L., Stump, A.: SMT-COMP: Satisfiability Modulo Theories Competition. In Etessami, K., Rajamani, S., eds.: 17th International Conference on Computer Aided Verification, Springer (2005) 20-23

7. Stump, A., Barrett, C.W., Dill, D.L., Levitt, J.: A Decision Procedure for an Extensional Theory of Arrays. In: Proceedings of LICS-16, IEEE (2001)

8. Armando, A., Ranise, S., Rusinowitch, M.: A Rewriting Approach to Satisfiability Procedures. Information and Computation 183 (2003) 140-164

9. Armando, A., Bonacina, M.P., Ranise, S., Schulz, S.: On a rewriting approach to satisfiability procedures: Extension, combination of theories and an experimental appraisal. In Gramlich, B., ed.: Proceedings of FroCoS. Volume 3717 of Lecture Notes in Computer Science., Springer (2005) 65-80 
10. Cyrluk, D., Möller, O., Rueß, H.: An Efficient Decision Procedure for the Theory of Fixed-Sized Bit-Vectors. [12] 60-71

11. Barrett, C.W., Dill, D.L., Levitt, J.R.: A decision procedure for bit-vector arithmetic. In: Proceedings of DAC. (1998) 522-527

12. Grumberg, O., ed.: Computer Aided Verification. 9th International Conference (CAV97). Haifa, Israel, June 22-25, 1997: Proceedings. Volume 1254 of Lecture Notes in Computer Science LNCS., Berlin - Heidelberg - New York, Springer (1997)

13. Möller, O., Rueß, H.: Solving Bit-Vector Equations. In Gopalakrishnan, G., Windley, P., eds.: Formal Methods in Computer-Aided Design (FMCAD '98). Volume 1522 of Lecture Notes in Computer Science., Palo Alto, CA, Springer-Verlag (1998) $36-48$

14. Bozzano, M., Bruttomesso, R., Cimatti, A., Franzen, A., Hanna, Z., Khasidashvili, Z., Palti, A., Sebastiani, R.: Encoding RTL Constructs for MATHSAT: a Preliminary Report. In Armando, A., Cimatti, A., eds.: the 3rd International Workshop on Pragmatics of Decision Procedures in Automated Reasoning (PDPAR 2005). (2005) To appear on the Electronic Notes in Theoretical Computer Science.

15. Clarke, E., Kroening, D., Yorav, K.: Behavioral Consistency of C and Verilog Programs. Technical Report CMU-CS-03-126, Computer Science Department, School of Computer Science, Carnegie Mellon University (2003)

16. Moskewicz, M.W., Madigan, C.F., Zhao, Y., Zhang, L., Malik, S.: Chaff: Engineering an Efficient SAT Solver. In: Proceedings of DAC, ACM (2001) 530-535

17. Knuth, D.: The Art of Computer Programming, Volume 3: Sorting and Searching. Volume 3. Addison-Wesley (1997)

18. Bellman, R.E.: On a Routing Problem. Quarterly of applied mathematics 16 (1958) 87-90

19. Ford, L.R., Fulkerson, D.R.: Flows in Networks. Princeton University Press (1962)

20. Prim, R.C.: Shortest Connection Networks and Some Generalisations. Bell System Technical Journal 36 (1957) 1389-1401

21. Stump, A., Barrett, C.W., Dill, D.L.: CVC: a Cooperating Validity Checker. In Brinksma, E., Larsen, K.G., eds.: Proceedings of CAV. Volume 2404 of LNCS., Springer (2002)

22. Ball, T., Rajamani, S.K.: Automatically Validating Temporal Safety Properties of Interfaces. In: Proceedings of SPIN, Springer New York, Inc. (2001) 103-122

23. Henzinger, T., Jhala, R., Majumdar, R., Sutre, G.: Software Verification with Blast. In Ball, T., Rajamani, S.K., eds.: Proceedings of SPIN. Volume 2648 of LNCS., Springer (2003) 235-239

24. Chaki, S., Clarke, E., Groce, A., Jha, S., Veith, H.: Modular Verification of Software Components in C. In: Proceedings of ICSE, IEEE Computer Society (2003) 385395

25. Xie, Y., Aiken, A.: Scalable error detection using boolean satisfiability. In Palsberg, J., Abadi, M., eds.: Proceedings of POPL, ACM Press (2005) 351-363 